\title{
Synthesis and characterization of SPIO-loaded PEG- $b$-PS micelles as contrast agent for long-term nanoparticle-based MRI phantom
}

\author{
MAN THEERASILP ${ }^{1,2}$, WITAYA SUNGKARAT ${ }^{3}$ and NORASED NASONGKLA ${ }^{1,2, *}$ \\ ${ }^{1}$ Department of Biomedical Engineering, Faculty of Engineering, Mahidol University, Phutthamonthon, Nakhon Pathom \\ 73170, Thailand \\ ${ }^{2}$ Department of Chemistry and Center of Excellence for Innovation in Chemistry, Faculty of Science, Mahidol University, \\ Bangkok 10400, Thailand \\ ${ }^{3}$ Department of Radiology, Faculty of Medicine, Ramathibodi Hospital, Mahidol University, Bangkok 10400, Thailand \\ *Author for correspondence (norased.nas@mahidol.ac.th)
}

MS received 30 January 2017; accepted 12 July 2017; published online 27 March 2018

\begin{abstract}
Nanoparticle-based magnetic resonance imaging (MRI) phantom was developed from the suspension of magnetic nanoparticles composing methoxy poly(ethylene glycol)-block-poly(styrene) (PEG- $b$-PS) micelles and superparamagnetic iron oxide (SPIO) nanoparticles in the core of micelles. The size of SPIO-loaded micelles was determined by dynamic light scattering (DLS) and transmission electron microscopy. Larger-size micelles were found when SPIO loading was increased. The effect of the hydrophobic section of block copolymer on the size of micelles was studied by DLS. Transverse relaxivity was evaluated on both 1.5 and $3 \mathrm{~T}$ clinical MRI scanner. Higher SPIO-loading provided higher relaxivity, where the relaxivity of $10 \%$ SPIO-loaded PEG(5 kDa)- $b$-PS(5 kDa) were 144.0 and $174.0 \mathrm{~m} \mathrm{M}^{-1} \mathrm{~s}^{-1}$ for 1.5 and $3 \mathrm{~T}$ MRI, respectively. This formulation showed stability over a 10-week period, and the standard deviations of the relaxivities were 3.0 and $8.0 \%$ for 1.5 and 3 T MRI, respectively. Thus, SPIO-loaded PEG- $b$-PS micelles have a potential to be applied as a contrast agent for nanoparticle-based MRI phantom.
\end{abstract}

Keywords. Iron oxide nanoparticle; polymeric micelles; stability; relaxivity; MRI phantom.

\section{Introduction}

Recently, non-invasive imaging techniques, especially magnetic resonance imaging (MRI), were developed to quantify the amount of iron in liver [1] and heart [2]. Thus, MRI phantoms are essential in calibrating MRI systems as well as in maintenance. An ideal material to make MRI phantom should provide uniform signal intensity and relaxation times in the range of human tissues or diseases. The material should be stable over a period of time and without signal interference [3]. Several transition metals were applied as paramagnetic contrast agents to prepare phantoms with desired $T_{1}$ and $T_{2}$ values such as nickel [4], gadolinium [3] and iron [5] compounds. Nevertheless, only one type of metal was developed as magnetic nanoparticle contrast agent, Ferumoxides-for MRI phantom [6]. However, these particles lack the reliable stability due to the precipitation of irons at the bottom of the tubes in the phantom [5].

Superparamagnetic iron oxide (SPIO) nanoparticles were composed of water insoluble iron oxides: magnetite $\left(\mathrm{Fe}_{3} \mathrm{O}_{4}\right)$, maghemite $\left(\mathrm{Fe}_{2} \mathrm{O}_{3}\right)$, or other types of ferrites. Under external magnetic field, SPIOs produce small magnetic fields which will shorten the relaxation time $\left(T_{1}, T_{2}\right.$ and $\left.T_{2^{*}}\right)$ of the surrounding water protons. This is called proton relaxation enhancement $-\mathrm{a}$ change of nuclear magnetic resonance (NMR) signal of the surrounding area. SPIO is used for $T_{2}$-weighed MRI by the shortening of $T_{2}$. In addition, the magnetic field of SPIO can affect a large number of surrounding water molecules. Therefore, MRI contrast can be increased by using SPIO as a $T_{2}$ contrast enhancing agent. Many coating materials were applied for SPIO to control the shape and size of particles, or to be used as the template for synthesis $[7,8]$. Polymeric micelles—one of the coating materials - are used to increase solubility $[9,10]$, enhance magnetic properties and improve biocompatibility of SPIO $[11,12]$. Polymeric micelles are spherically shaped nanoparticles with the size in the nanometer level. It composes of two segments each with different functions: (1) the inner core is made up of the hydrophobic segment, and (2) the outer shell is made up of a hydrophilic segment. The core acts as a reservoir for encapsulating drug to enhance solubility and increase drug stability $[13,14]$. Poly(ethylene glycol) (PEG) was widely used as the outer shell because of its excellent hydrophilic property. The amphiphilic block copolymer was commonly used to make polymeric micelles due to its capability of self-assembly in aqueous environment by hydrophobic interaction.

In this study, we report on the method of preparing nanoparticle-based MRI phantom using methoxy poly (ethylene glycol)-block-poly(styrene) to form SPIO-loaded 
micelles. The block copolymer was synthesized via atom transfer radical polymerization. SPIO was synthesized by thermal decomposition method. SPIO-loaded micelles were self-assembled in water by solvent evaporation method. Then, micelles were evaluated for their particles size, MR sensitivity and stability.

\section{Materials and methods}

\subsection{Materials}

Methoxy-terminated poly(ethylene glycol) (MeO-PEG) with a molecular weight (MW) of 5000 Dalton was purchased from Fluka and was purified by recrystallization with ethyl acetate. Triethylamine (TEA) and styrene were purchased from Sigma and purified by distillation over calcium hydride $\left(\mathrm{CaH}_{2}\right)$, and stored over the molecular sieve. Tetrahydrofuran (THF) was purchased from RCI Labscan and dried by refluxing over a sodium-potassium alloy and distilled under dry argon. Copper (I) bromide (CuBr) was purchased from Sigma, purified by stirring in glacial acetic acid overnight, and then centrifuged at $3000 \mathrm{rpm}$ for $10 \mathrm{~min}$; the supernatant was discarded, and washed with diethyl ether and absolute ethanol before drying overnight at $70^{\circ} \mathrm{C}$ under a vacuum. Iron (III) acetylacetonate $\left(\mathrm{Fe}(\mathrm{acac})_{3}\right), 1,2-$ hexadecanediol, oleic acid, oleylamine, benzyl ether, $\mathrm{N}$, $\mathrm{N}, \mathrm{N}^{\prime}, \mathrm{N}^{\prime \prime}, \mathrm{N}^{\prime \prime}$-pentamethyldiethylenetriamine (PMDETA) and $\alpha$-bromoisobutyryl bromide (BiBB) were purchased from Aldrich and used as received.

\subsection{Synthesis of PEG-b-PS}

2.2a Preparation of $\mathrm{MeO}-\mathrm{PEG}-\mathrm{Br}$ : MeO-PEG (2 g) was dried overnight at $65^{\circ} \mathrm{C}$ under a vacuum, dissolved in $20 \mathrm{ml}$ anhydrous THF, and then mixed with $279 \mu 1$ of triethyamine (5 molar equivalents). The solution was stirred for $30 \mathrm{~min}$ in a salted ice-water bath. Two hundred and forty-seven microlitres of BiBB (5 molar equivalents) was added dropwise to the mixture. The solution was stirred for $1 \mathrm{~h}$ in the salted ice-water bath, then for $48 \mathrm{~h}$ in room temperature under argon atmosphere. The crude product was centrifuged to remove precipitant. MeO-PEG-Br was precipitated in cold ethyl ether, purified by re-dissolving in acetone, precipitated in diethyl ether twice, and then dried under vacuum at room temperature.

2.2b Synthesis of MeO-PEG-b-PS: MeO-PEG-Br (1.5 g) in a $50 \mathrm{ml}$ two-neck round bottomed flask was dried overnight at $65^{\circ} \mathrm{C}$ under a vacuum. One hundred and twenty-two microlitres of PMDETA (2 molar equivalents) was added and the mixture was degassed; then $\mathrm{CuBr}$ was added and the mixture was degassed again. The mixture was stirred and heated at $110^{\circ} \mathrm{C}$ in an oil bath for $20 \mathrm{~h}$ under argon atmosphere; and the mixture was quickly cooled to room temperature with cold water. Twenty microlitres of THF was added into the crude product and centrifuged to remove precipitant, and then the crude product was precipitated in cold hexane and centrifuged. The solid precipitant was then dissolved in THF and dropped into water under ultrasonic. The solution was dialysed against deionized water (DI) for 2 days (MW cut-off: $50 \mathrm{kDa}$ ) and the resulting suspension was freeze-dried. The polymer structure was investigated by ${ }^{1} \mathrm{H}-\mathrm{NMR}$ spectra. The number-average molecular weight $\left(M_{\mathrm{n}}\right)$ of the copolymer was determined from ${ }^{1} \mathrm{H}$-NMR integral peak ratio and gel permeation chromatography (GPC).

\subsection{Synthesis of SPIO}

SPIO was synthesized according to the Sun et al's method [15]. Briefly, $\mathrm{Fe}(\mathrm{acac})_{3}$ (2 mmol), 1,2-hexadecanediol (10 mmol), oleic acid (6 mmol), oleylamine $(6 \mathrm{mmol})$ and benzyl ether $(20 \mathrm{ml})$ were mixed in a reaction flask by magnetic stirring under nitrogen flow. The mixture was then heated to $200^{\circ} \mathrm{C}$, reacted for $2 \mathrm{~h}$, and finally refluxed at $300^{\circ} \mathrm{C}$ for another $1 \mathrm{~h}$. The black mixture was cooled to room temperature and ethanol was added to precipitate SPIO; then centrifuged at $4000 \mathrm{rpm}$ for $10 \mathrm{~min}$ at room temperature. SPIO was re-dissolved in hexane, precipitated into ethanol, and centrifuged at $4000 \mathrm{rpm}$ for $10 \mathrm{~min}$ at room temperature. The black-brown nanoparticles were dissolved in hexane for storage prior to use.

\subsection{Preparation of SPIO-loaded micelles}

For 5\% SPIO-micelles, $5.0 \mathrm{mg}$ of dry-weight SPIO and $95.0 \mathrm{mg}$ of PEG- $b$-PS were dissolved in THF and then dropped into $20 \mathrm{ml}$ of DI water, while sonicating. The solution was gently stirred to allow THF to evaporate slowly at room temperature. Micelles can be formed during the evaporation of THF. SPIO-micelles were filtered by $0.45 \mu \mathrm{m}$ syringe filter. Centrifugal filter was used to concentrate SPIO-micelles. For $10 \%$ SPIO-micelles, $10 \mathrm{mg}$ of dry-weight SPIO and $90 \mathrm{mg}$ of polymer were dissolved in THF; then it was used for micelle preparation.

\subsection{Determination of iron concentration}

$37 \% \mathrm{HCl}(1 \mathrm{ml})$ was added into $100 \mu \mathrm{l}$ of SPIO-micelles. The mixture was incubated at $60^{\circ} \mathrm{C}$ for $12 \mathrm{~h}$; then the solution was transferred to $10 \mathrm{ml}$ volumetric flask and DI water was added. Fe concentration was determined by atomic absorption spectroscopy.

\section{$2.6 M R I$}

MRI measurement was performed on a clinical MRI scanner 1.5 and $3.0 \mathrm{~T}$ at room temperature. $T_{2}$ values were determined from exponential decay as shown in equation (1). Signal intensity and echo time are termed as SI and TE, respectively. Transverse relaxivity $\left(r_{2 *}\right)$ was determined by equation (2) 


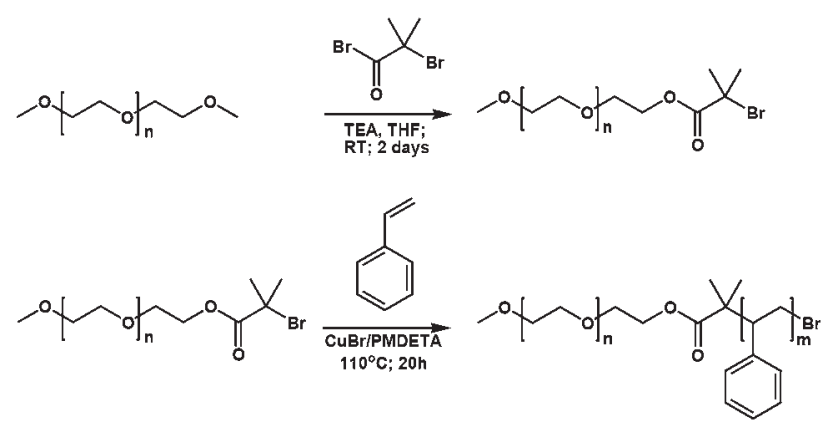

Figure 1. Synthesis route of MeO-PEG- $b$-PS.

from the slope of line regression from the plot of $1 / T_{2} * v s$. Fe concentration.

$$
\begin{aligned}
& S I_{\mathrm{TE}}=S I_{\mathrm{TE}=0} \exp \left[\frac{-T E}{T_{2}^{*}}\right], \\
& \frac{1}{T_{2}^{*}}=\frac{1}{T_{22_{2} \mathrm{H}_{2} \mathrm{O}}^{*}}+r_{2^{*}}[\mathrm{Fe}] .
\end{aligned}
$$

\section{Results and discussion}

\subsection{Characterization of PEG-b-PS}

The synthesis route of PEG- $b$-PS is outlined in figure 1 . The telechelic macroinitiators were prepared when the hydroxyl group of $\mathrm{MeO}-\mathrm{PEG}$ reacts with acid bromide, $\mathrm{BiBB}$. The reaction was carried out at $0^{\circ} \mathrm{C}$ to avoid the cleavage of $\mathrm{PEG}$ chain [16]. The substitution of the hydroxyl groups was confirmed by ${ }^{1} \mathrm{H}-\mathrm{NMR}$. The singlet peak of methoxy protons of PEG $\left(\mathrm{CH}_{3}-\mathrm{O}-\right)$ and methylene protons of PEG $\left(-\mathrm{OCH}_{2} \mathrm{CH}_{2} \mathrm{O}-\right)$ were assigned at 3.35 and $3.6 \mathrm{ppm}$, respectively. After substitution reaction, the chemical shift of products were found at $1.92\left(-\mathrm{C}-\mathrm{CH}_{3}\right)$ and $4.3\left(-\mathrm{CH}_{2}-\mathrm{O}-\mathrm{C}=\mathrm{O}\right)$. The polymer yield of PEG-Br was $87 \%$. The conversion rate of hydroxyl to bromo-functional group was calculated based on NMR spectra analysis, which was determined by integral values of absorption peaks $a, c$ and $d$ (2:3:6 ratio) in figure $2 \mathrm{a}$. The conversion rate of PEG into PEG-Br was calculated as

$$
(9 / 2) \times[c /(a+d)] \times 100 \% .
$$

So, the conversion rate was $93.8 \%$. PEG-b-PS was synthesized by atom transfer radical polymerization (ATRP), where a copper complex compound (transition metal compound) acts as a carrier of bromine (halogen atom) at the end of PEG in a reversible redox process. After styrene copolymerization, the signal of the phenyl protons appeared at $6.30-7.30 \mathrm{ppm}$ (peak g's region in figure $2 b$ ), and the methylene protons appeared at 1.43-1.72 ppm (peaks e and $\mathrm{f}$ in figure $2 \mathrm{~b}$ ). The MW of polystyrene was $5.4 \mathrm{kDa}$ determined by comparing ${ }^{1} \mathrm{H}-\mathrm{NMR}$ integral signal intensity between methylene proton of PEG at $3.6 \mathrm{ppm}$ and phenyl protons of PS between 6.30 and $7.30 \mathrm{ppm}$. Therefore, the MW of PEG- $b$-PS was $10.4 \mathrm{kDa}$ [17]. From GPC analysis, the polydispersity index of EG$b$-PS was 1.29 . The yield of PEG- $b$-PS was $76.1 \%$. These results indicate that PEG- $b$-PS was synthesized successfully by ATRP.

\subsection{Characterization of SPIO}

SPIO was synthesized by thermal decomposition method. This method provides a hydrophobic SPIO, because the surface of SPIO was coated by oleic acid and oleylamine with carbonyl and amine functional groups adsorbed to SPIO particles, respectively. This leads to long hydrophobic tails covered on the SPIO surface [18]. The size of the oleic acidand oleylamine-coated SPIO particles was $6.91 \pm 0.05 \mathrm{~nm}$ determined by DLS. Figure 3a shows the histogram of size measurement which had narrow distribution in size.

\subsection{Characterization of SPIO-loaded micelles}

SPIO was encapsulated into micelles by hydrophobic interactions to the polystyrene section in the block copolymer. Solvent evaporation method was utilized to fabricate SPIOloaded PEG- $b$-PS micelles. The micelle size was determined by DLS as shown in figure 3, and tables 1 and 2. Results showed that the micelles size increased when SPIO loading increased (figure 3). Interestingly, at the same SPIO loading, the size of the micelles for high-MW PS (5 kDa) were smaller than low-MWPS $(0.75 \mathrm{kDa})$. This is because of the hydrophobic section of $0.75 \mathrm{kDa}$ PS provides weak hydrophobic interaction that is unable to form stable micelles, resulting in the loss in packing at the core of micelles. It should be noted that the precipitate was found after a couple of weeks. Results are similar to our previous work, the determination of critical micelles concentration (CMC) of polymeric micelles with varied MWs of the hydrophobic chain [19]. The CMC of PEG- $b$-PLA increased when the MW of PLA decreased. The encapsulation capacity of micelles depends on the MW of the hydrophobic block. The high MW of PS in amphiphilic block copolymer increases the degree of hydrophobicity of micelles that results in the increase in their hydrophobic interaction to the encapsulated hydrophobic particles [20]. The micelle size increased considerably when the SPIO loading for PEG(5 kDa)- $b$-PS(0.75 kDa) micelles increased as shown in figure $3 b$; however, the micelle size increased slightly when the SPIO loading of PEG(5 kDa)$b$-PS $(5 \mathrm{kDa})$ micelles increased as shown in figure $3 \mathrm{c}$. The cluster formation of low- and high-MW polymers containing SPIO is quite different [21]. For low-MW polymer, clustering was driven by the attractive van der Waals force of each SPIO particle. Consequently, the clustering size is relative to the amount of loaded-SPIO. Whereas, high-MW polymer formed clustering by bridging SPIO particles with polymers [22], resulting in the effect of MW polymer on clustering size being more predominant than the amount of loaded-SPIO. 
(a)

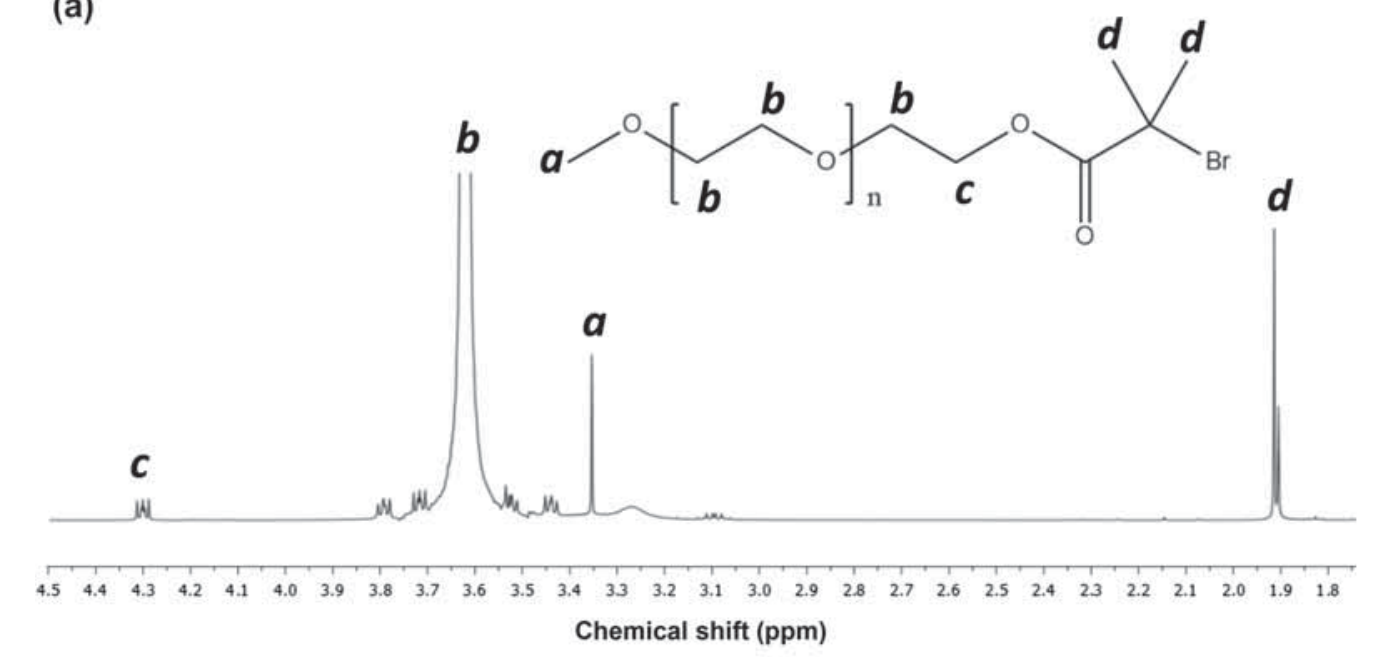

(b)

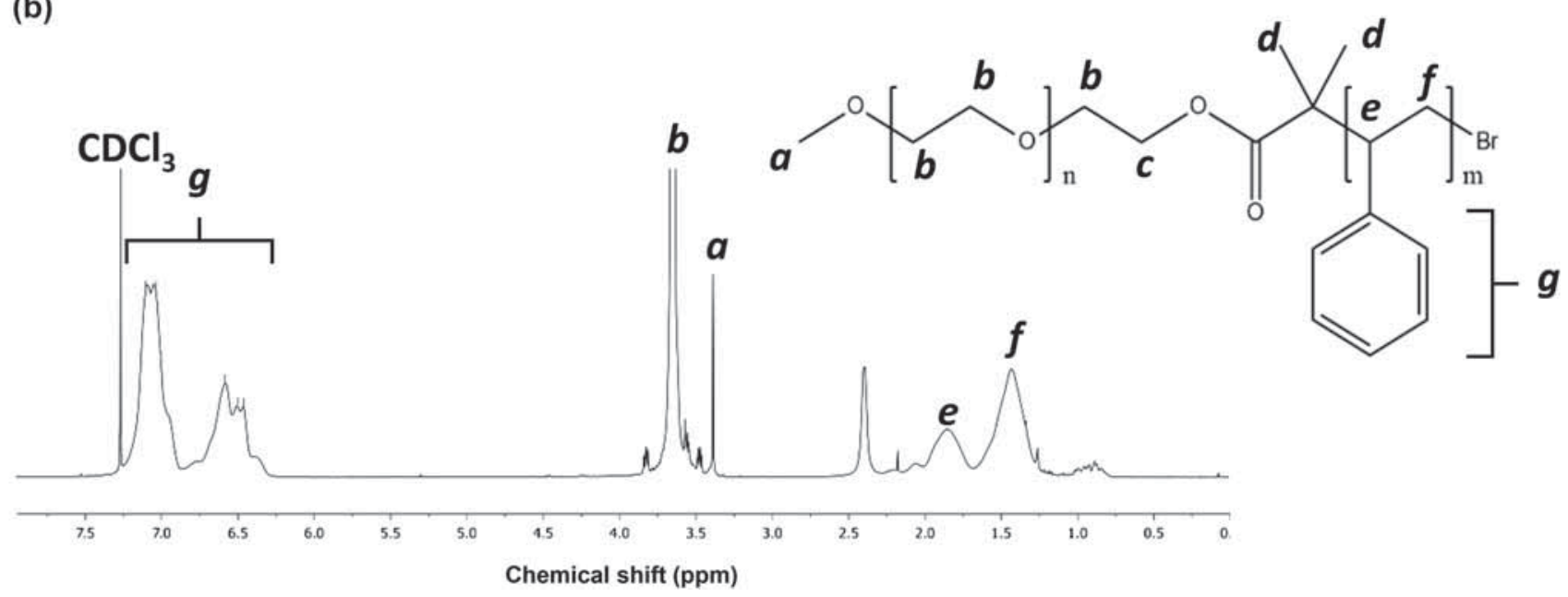

Figure 2. ${ }^{1} \mathrm{H}-\mathrm{NMR}$ spectra of (a) PEG-Br and (b) PEG-b-PS.

Therefore, the slight increase in the clustering size of SPIO-loaded high-MW PS micelles was found when increasing the amount of loaded-SPIO. TEM images shown in figure $4 \mathrm{a}, \mathrm{c}$ and e indicated that the size of the hydrophobicSPIO, 5\%- and 10\%-SPIO-loaded PEG(5 kDa)- $b$-PS(5 kDa) micelles were $5.1 \pm 0.6,26.1 \pm 5.5$ and $36.6 \pm 7.8 \mathrm{~nm}$, respectively. The size obtained from TEM is smaller than that of DLS since polymers coated on the surface of micelles have the electron transparent property and cannot be visualized by TEM [23]. Furthermore, TEM provides the size of micelles in dried state, while DLS measures the size in aqueous solution where the corona is hydrated later, resulting in an increase in the hydrodynamic volume [24]. Therefore, micelles size in wet state from DSL technique is bigger in comparison with the size in dry state from TEM. These results confirmed that micelles with $0.75 \mathrm{kDa}$ PS was not enough to encapsulate high SPIO loading, while micelles with $5 \mathrm{kDa}$ PS were stable up to $10 \%$ SPIO loading. There are two contributing reasons explaining the lack of stability of the low-MW PS micelles containing SPIO. First, the loading capacity of micelles is low. The loading capacity directly related to the size of hydrophobic core, where the loading capacity decreased when the MW of core micelles decreased [25]. Consequently, the unstable SPIO-micelles may occur from overloading of SPIO in low-MW PS micelles. Second, the high CMC is a result of the low-MW of PS block that occurs with the decrease of hydrophobic driving force for self-assembly micelles $[19,26]$.

Magnetic studies were carried out using a vibrating sample magnetometer (LakeShore Model 7404) at room temperature. Figure $4 \mathrm{~b}, \mathrm{~d}$ and e showed the magnetization loops of hydrophobic-SPIO, 5- and 10\%-SPIO-loaded PEG(5 kDa)-b-PS(5 kDa) micelles, respectively. All samples showed typical superparamagnetic behaviour, where the net magnetization returned to zero in the absence of an external field. A saturation magnetization $\left(M_{\mathrm{s}}\right)$ value of the hydrophobic-SPIO nanoparticles was $55.5 \mathrm{Fe} \mathrm{emu}^{-1}$. The $M_{\mathrm{s}}$ value was $56.1 \mathrm{Fe}^{\mathrm{emu}} \mathrm{g}^{-1}$ for $5 \%$-SPIO-loaded PEG(5 $\mathrm{kDa})-b$-PS $(5 \mathrm{kDa})$ and $59.4 \mathrm{Fe} \mathrm{emu} \mathrm{g}^{-1}$ for $10 \%$-SPIOloaded PEG(5 kDa)- $b$-PS(5 kDa). These results showed no 

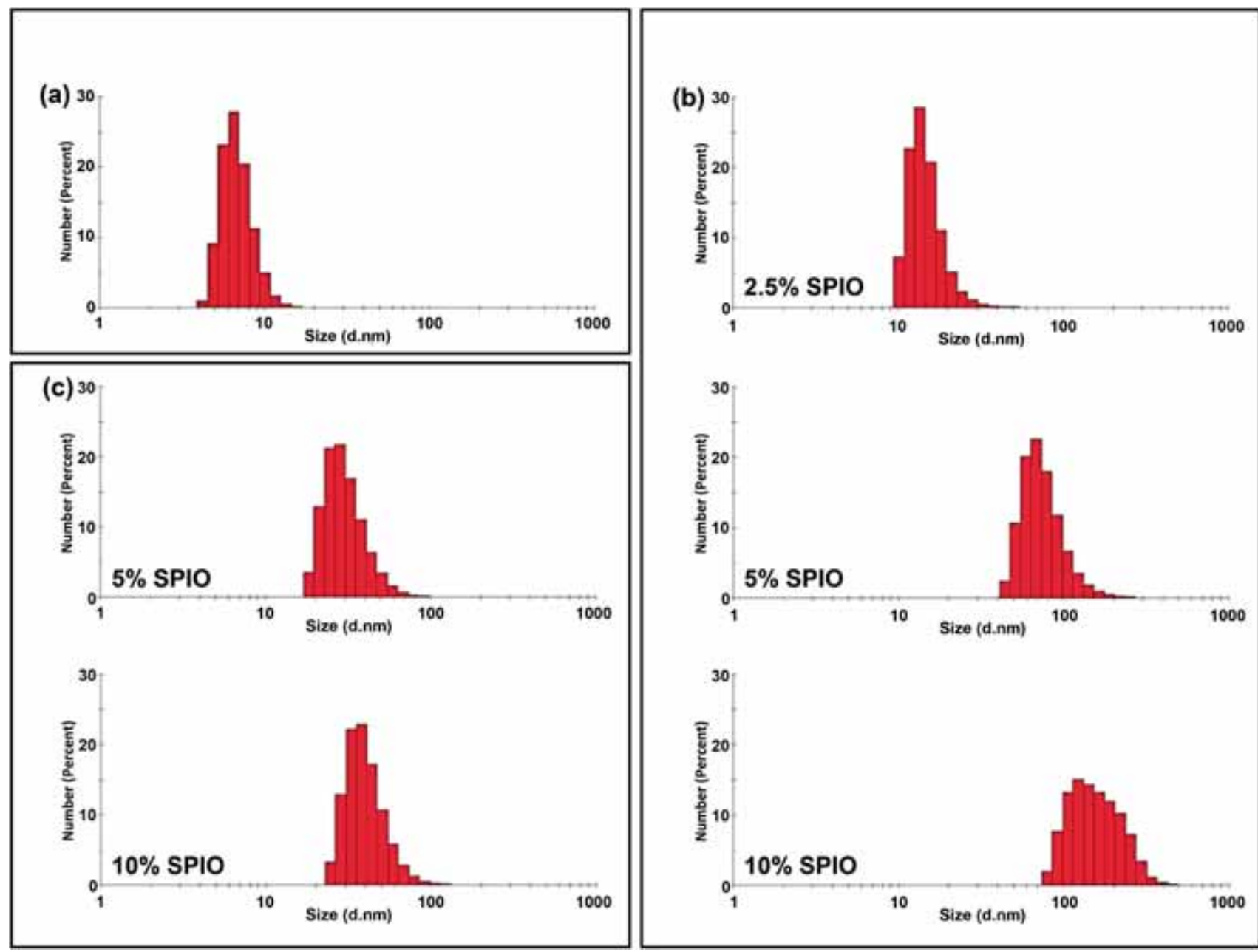

Figure 3. Dynamic light scattering histogram of (a) SPIO in hexane, (b) SPIO-loaded PEG ( 5 kDa)- $b$-PS(0.75 kDa) and (c) SPIO-loaded $\operatorname{PEG}(5 \mathrm{kDa})-b-\operatorname{PS}(5 \mathrm{kDa})$.

Table 1. Micelles size and relaxivity $\left(r_{2}\right)$ of SPIO-loaded PEG(5 kDa)- $b$-PS( $\left.0.75 \mathrm{kDa}\right)$ micelle.

\begin{tabular}{lcc}
\hline Formulation & Micelles size $^{\mathrm{a}}(\mathrm{nm})$ & Relaxivity $^{\mathrm{b}}, r_{2}\left(\mathrm{~m} \mathrm{M}^{-1} \mathrm{~s}^{-1}\right)$ \\
\hline 2.5\% SPIO, PEG(5 kDa)- $b$-PS(0.75 kDa) & $14.3 \pm 0.6$ & 97.6 \\
$5 \%$ SPIO, PEG(5 kDa)- $b$-PS $(0.75 \mathrm{kDa})$ & $77.8 \pm 4.9$ & 136.1 \\
$10 \%$ SPIO, PEG(5 kDa)- $b$-PS(0.75 kDa) & $156.0 \pm 7.3$ & 188.0 \\
\hline
\end{tabular}

${ }^{\mathrm{a}}$ Measured by DLS; ${ }^{\mathrm{s}}$ scanning on 1.5 T MRI.

Table 2. Micelles size, saturation magnetization $\left(M_{\mathrm{S}}\right)$ and relaxivity $\left(r_{2}\right)$ of hydrophobic SPIO and SPIO-loaded micelle PEG(5 kDa)- $b$-PS(5 kDa).

\begin{tabular}{|c|c|c|c|c|c|}
\hline \multirow[b]{2}{*}{ Formulation } & \multirow[b]{2}{*}{$M_{\mathrm{S}}\left(\mathrm{emu} \mathrm{g}^{-1} \mathrm{SPIO}\right)$} & \multicolumn{2}{|c|}{ Micelles size (nm) } & \multicolumn{2}{|c|}{ Relaxivity, $r_{2^{*}}\left(\mathrm{~m} \mathrm{M}^{-1} \mathrm{~s}^{-1}\right)$} \\
\hline & & DLS & TEM & $1.5 \mathrm{~T}$ MRI & $3 \mathrm{~T}$ MRI \\
\hline Hydrophobic SPIO & 55.5 & $6.91 \pm 0.05$ & $5.1 \pm 0.6$ & - & - \\
\hline $5 \%$ SPIO, PEG(5 kDa)- $b-\mathrm{PS}(5 \mathrm{kDa})$ & 56.1 & $29.9 \pm 2.8$ & $26.1 \pm 5.5$ & 82.8 & 93.0 \\
\hline $10 \%$ SPIO, PEG(5 kDa)- $b$-PS( $5 \mathrm{kDa})$ & 59.4 & $40.8 \pm 1.3$ & $36.6 \pm 7.8$ & 144.0 & 174.4 \\
\hline
\end{tabular}



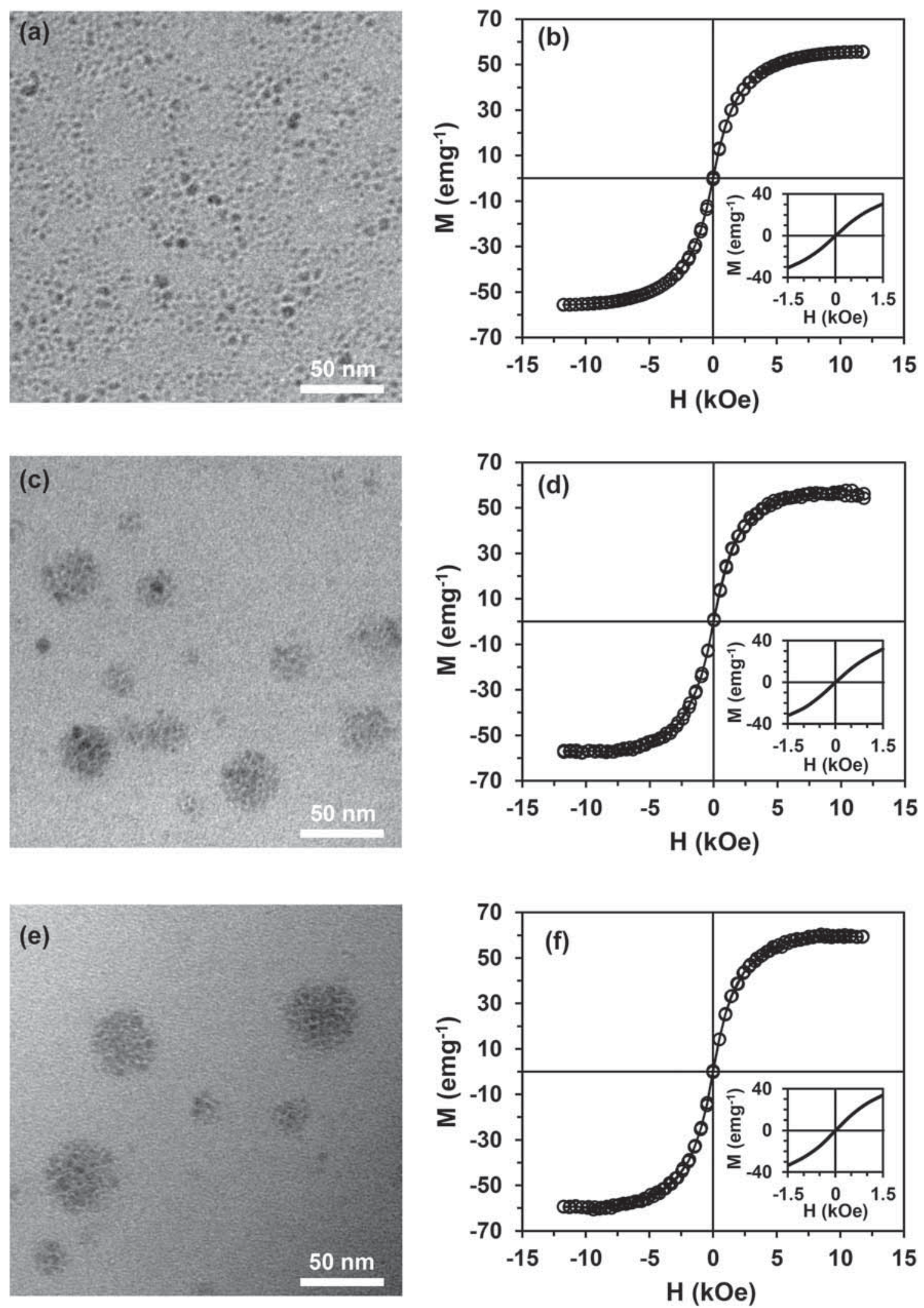

Figure 4. Transmission electron microscopy (TEM) images of SPIO (a), 5\% (c) and 10\% (e) SPIO-loaded PEG(5 kDa)$b$-PS(5 kDa). Hysteresis loops of SPIO (b), 5\% (d) and 10\% (f) SPIO-loaded PEG(5 kDa)- $b$-PS(5 kDa) at room temperature. The inset shows a zoomed-in plot between -1.5 and $1.5 \mathrm{kOe}$ magnetic field. 

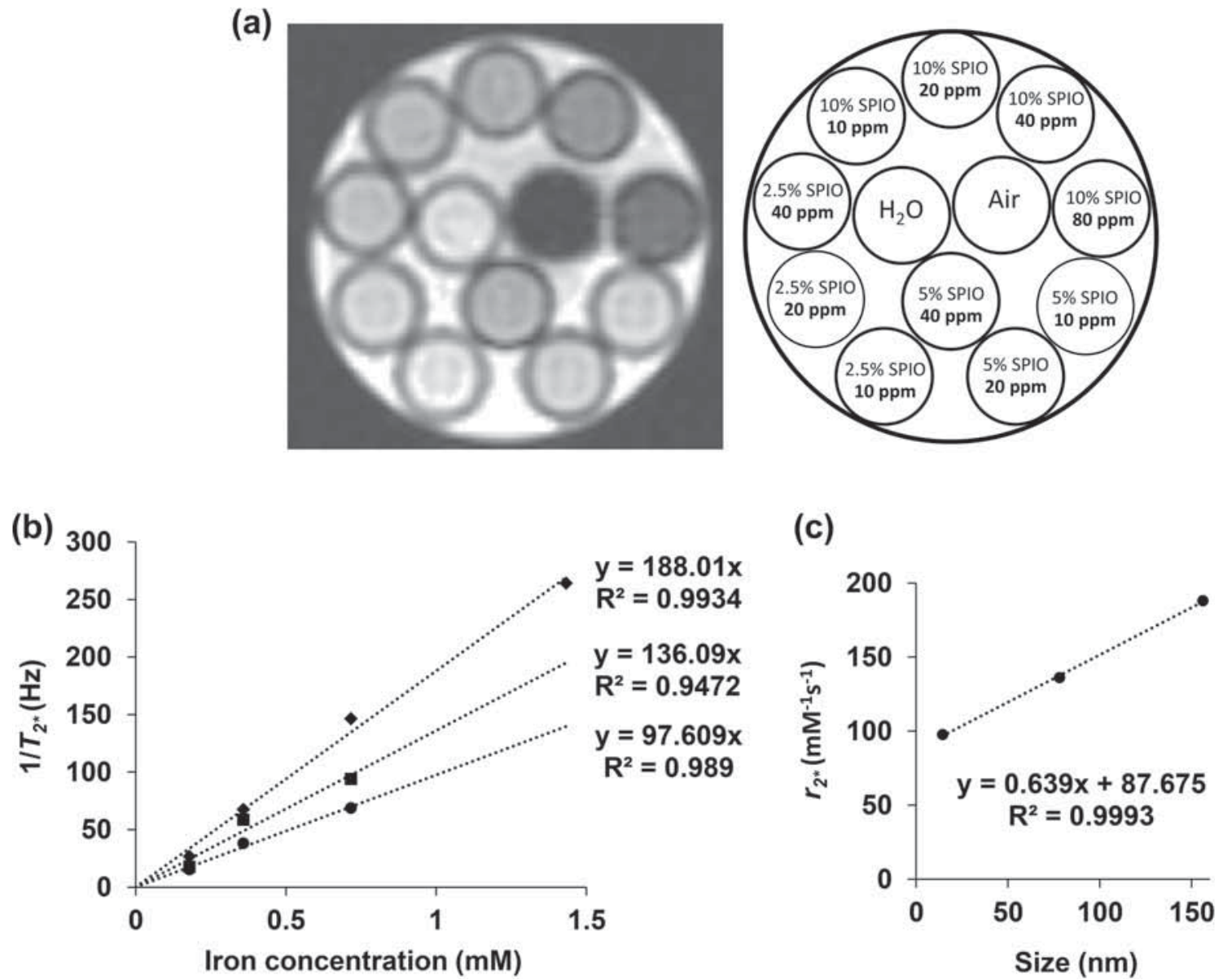

Figure 5. (a) $T_{2}$-weighted images of SPIO-loaded PEG(5 kDa)- $b$-PS( $(0.75 \mathrm{kDa})$ micelles at different iron concentrations measured by a $1.5 \mathrm{~T}$ MRI. (b) Relaxation rate (Hz) of $T_{2 *}$ as a function of iron concentration (ppm) for $2.5 \%(\bullet)$, $5 \%(\boldsymbol{\square})$ and $10 \%$ ( SPIO-loaded PEG- $b$-PS micelles. Dashed line represents the linear regression. (c) Dependency of the relaxivity on cluster size of the SPIO micelles. Dashed line represents the linear regression.

obvious loss in magnetization per $\mathrm{Fe}$ unit. Similar phenomenon was reported for SPIO-loaded poly(ethylene glycol) and poly( $\varepsilon$-caprolactone) micelles [27]. These results suggest that clustering size and the amount of SPIO loading in polymeric micelles do not affect the magnetization behaviours of SPIO.

\subsection{MRI and relaxivity}

$T_{2^{*}-\text { weighed MRI of SPIO-loaded micelles at a } 1.5 \mathrm{~T} \text { clinical }}$ MRI scanner is shown in figure $5 \mathrm{a}$ and table 1. SPIO-loaded micelles exhibited negative contrast enhancement as the concentration increased. The relaxation rate $\left(1 / T_{2^{*}}\right)$ was linearly proportional to the Fe concentration of SPIO as shown in figure $5 \mathrm{~b}$. The efficiency of the negative contrast agent was defined by the relaxivity $\left(r_{2 *}\right)$, whereby higher $r_{2 *}$ values indicate higher quality, because smaller amount of SPIO produces larger magnetization and exhibits high relaxivity. The $r_{2^{*}}$ values were determined from the slope of the plot between $1 / T_{2^{*}}$ and Fe concentration. In the case of a single particle, magnetization or relaxivity generated by magnetic domains in SPIO particles depends on the size of SPIO. The SPIO particles with bigger size produce higher magnetization, but the size should not exceed $20 \mathrm{~nm}$, which is the limitation of a single SPIO particle [28]. Unlike single SPIO particle, for SPIO-loaded micelles, higher relaxivity can be produced when SPIO loading increased from a so-called 'the clustering effect'. Furthermore, many reports showed that MR signal was significantly increased by the clustering effect because of enhanced magnetic properties [12,27,29,30]. According to the outer sphere relaxation theory, the $r_{2 *}$ value depends on the cluster size [31]. The $r_{2 *}$ value was divided into three regimes through the increase in cluster size. First, the motional averaging regime (MAR) is where the relaxivity increased with the increasing size of SPIO cluster. However, the relaxivity reaches its maximum in the static dephasing regime (SDR); then, it decreased with increasing clustering size in the echolimiting regime (ELR).

SPIO-loaded PEG- $b$-PS micelles presented similar phenomena in MAR. The $r_{2^{*}}$ values were linearly increased by 

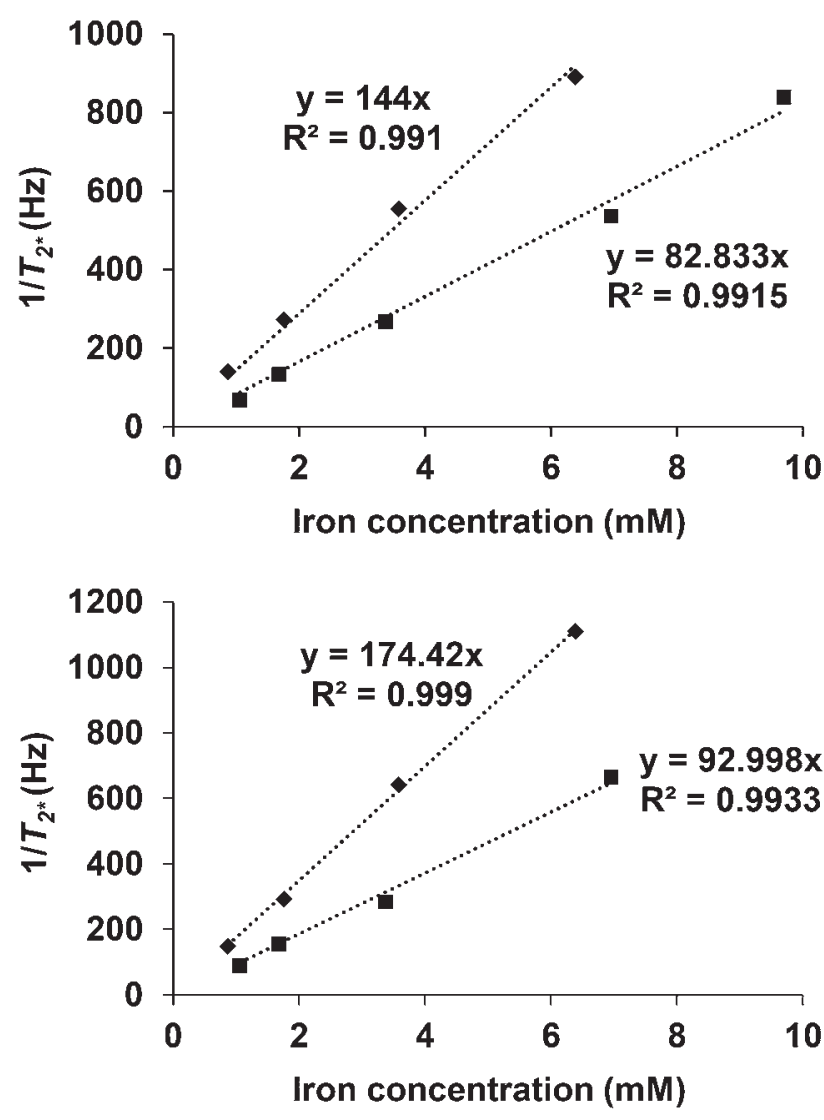

Figure 6. Relaxation rates $(\mathrm{Hz})$ of $T_{2} *$ as a function of iron concentration $(\mathrm{mM})$ for $5 \%(\boldsymbol{\square})$ and $10 \%(\checkmark)$ SPIO-loaded PEG(5 kDa)$b$-PS( $5 \mathrm{kDa}$ ) micelles measured by a $1.5 \mathrm{~T}$ (above) and $3 \mathrm{~T}$ (below) MRI scanner at room temperature. Dashed line represents the linear regression.

(a)

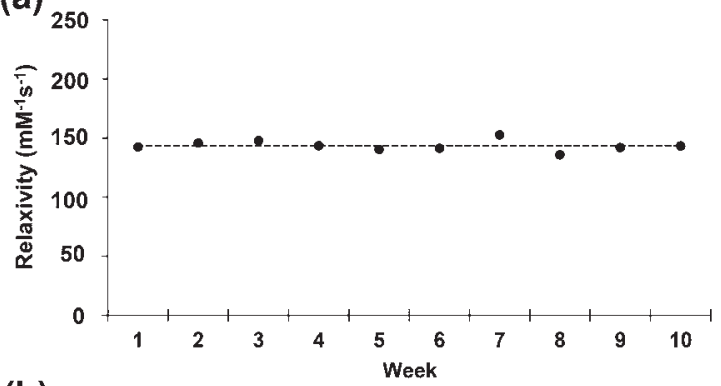

(b)

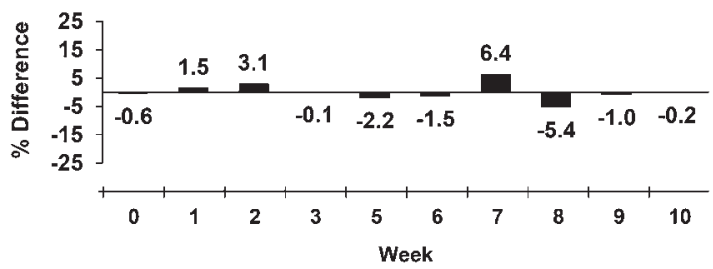

the size of micelles, where the values were 97.6, 136.1 and $188.0 \mathrm{~m} \mathrm{M}^{-1} \mathrm{~s}^{-1}$ with the micelle size of 14,78 and $188 \mathrm{~nm}$, respectively, as shown in figure $5 \mathrm{c}$. The relaxivity of SPIOloaded PEG(5 kDa)- $b$-PS( $5 \mathrm{kDa})$ micelles was determined as shown in figure 6 and table 2. For $1.5 \mathrm{~T}$ MRI, the relaxivity of 5 and $10 \%$ SPIO-loading was 82.8 and $144.0 \mathrm{~m} \mathrm{M}^{-1} \mathrm{~s}^{-1}$, respectively. For 3 T MRI, the relaxivity of 5 and $10 \%$ SPIOloading was 93.0 and $174.4 \mathrm{~m} \mathrm{M}^{-1} \mathrm{~s}^{-1}$, respectively. These results suggested that 5 and 10\% SPIO-loading in high-MW PS micelles were in MAR - the same as low-MW PS micelles. It should be noted that the relaxivity of high-MW PS block was lower than that of low-MW PS block. This was due to an increase in the hydrophobic property of the core of PS micelles. This led to the prevention of water protons from contacting the SPIO, resulting in a decrease in relaxivity. These results are similar to the previous report that showed the dependence of relaxivity on coating thickness and hydrophilicity of coating materials [32].

\subsection{Stability test of SPIO-loaded micelles}

DLS technique [33] and MR signal intensity [34] were used to evaluate the stability of SPIO nanoparticles. For this work, the MR signal intensity was selected to investigate the stability of SPIO micelles by measuring its relaxivity $\left(r_{2^{*}}\right)$; this is because using MR signal has advantages over other methods. First, all SPIO concentrations were calculated resulting in a more accurate data. Second, the MR sensitivity of SPIO was monitored during the course of the experiment. Finally, the relaxivity value is the direct measurement of MR signal stability, while DLS, which is the indirect method, resulting in the variation of MR signal because aggregation may change relaxivity

(c)

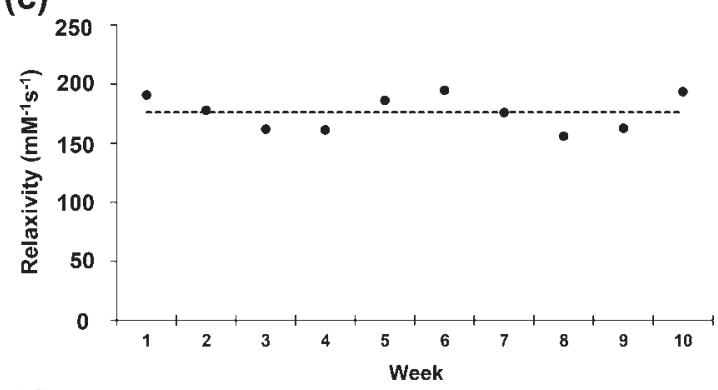

(d)

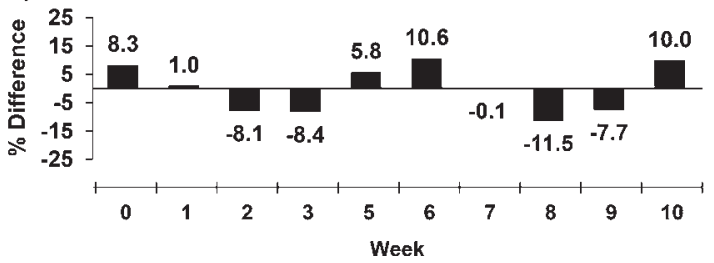

Figure 7. Relaxivity of $10 \%$ SPIO-loaded micelles PEG(5 kDa)- $b$-PS( $5 \mathrm{kDa})$ in water over 10 weeks. Measured on a $1.5 \mathrm{~T}(\mathbf{a}, \mathbf{b})$ and $3 \mathrm{~T}(\mathbf{c}, \mathbf{d})$ MRI scanner at room temperature. Dashed line represents average value. Percentage difference against mean relaxivity over 10 weeks measured by $1.5 \mathrm{~T}$ (b) and $3 \mathrm{~T}$ (d) MRI scanner. 
during aggregation process - is used to monitor nanoparticles aggregation [35].

Relaxivity was determined every week during the course of experiment. The average and standard deviation of relaxivity were calculated from every data point of each formulation. For 1.5 T MRI scanner, the average relaxivity of $10 \%$ SPIO-loaded micelles and its standard deviation were $144 \mathrm{~m} \mathrm{M}^{-1} \mathrm{~s}^{-1}$ and $3.0 \%$ (figure $7 \mathrm{a}$ ), respectively. For $3 \mathrm{~T}$ MRI, the average relaxivity of $10 \%$ SPIO-loaded micelles was $176 \mathrm{~m} \mathrm{M}^{-1} \mathrm{~s}^{-1}$ and its standard deviation was $8.0 \%$ (figure 7c). The percentage difference with respect to the average value is shown in figure $7 \mathrm{~b}$ and $\mathrm{d}$. The maximum deviation was $6.4 \%$ at week 7 based on $1.5 \mathrm{~T}$ MRI (figure $7 \mathrm{~b}$ ). For $3 \mathrm{~T}$ MRI, the maximum deviation was $11.5 \%$ at week 8 (figure 7d). The higher SPIO-loading, 20\%-SPIO-loaded $\operatorname{PEG}(5 \mathrm{kDa})-b$-PS(5 $\mathrm{kDa})$ were also prepared to evaluate relaxivity. The relaxivity on $3 \mathrm{~T}$ MRI-scanner was $432 \mathrm{~m} \mathrm{M}^{-1} \mathrm{~s}^{-1}$. High relaxivity was found because of clustering effect of SPIO in micelles. Nevertheless, its stability of relaxivity was poor; the relaxivity decreased from 432 to $337 \mathrm{~m} \mathrm{M}^{-1} \mathrm{~s}^{-1}(\sim 22 \%)$ after 10 weeks (data not shown). These results indicated that $10 \%$-SPIO-loaded PEG(5 kDa)- $b$-PS(5 kDa) micelles is stable, and has high sensitivity and low deviation of relaxivity; thus, this formulation has a potential application as MRI phantom for $1 / T_{2^{*}}$ mapping.

\section{Conclusion}

A nanoparticle-based phantom for $1 / T_{2} *$ mapping applications with stable MR signal over a 10-week period was developed. Nanoparticles were composed of non-biodegradable amphiphilic block copolymer (PEG- $b$-PS) and cluster of superparamagnetic iron oxide nanoparticles (SPIO), where SPIOs were encapsulated in the core of micelles. SPIO-loaded micelles showed high relaxivity because of the clustering effect of SPIO in the core of micelles, where the relaxivity can be adjusted by changing the amount of SPIO loading. These results indicate that the magnetic nanoparticles encapsulated polymeric micelles have potential application as MRI phantom.

\section{Acknowledgements}

This research was supported by Mahidol University, Thailand.

\section{References}

[1] Alústiza Echeverría J M, Castiella A and Emparanza J I 2012 Insights Imaging $\mathbf{3} 173$

[2] Anderson L J, Holden S, Davis B, Prescott E, Charrier C C, Bunce N H et al 2001 Eur. Heart J. 222171
[3] Yoshimura K, Kato H, Kuroda M, Yoshida A, Hanamoto K, Tanaka A et al 2003 Magn. Reson. Med. 501011

[4] Vassiliou V S, Heng E L, Gatehouse P D, Donovan J, Raphael C E, Giri S et al 2016 J. Cardiovasc. Magn. Reson. 181

[5] Alústiza J M, Emparanza J I, Castiella A, Casado A, Garrido A, Aldazábal P et al 2015 Biomed Res. Int. 2015 Article no. 294024

[6] Ernst O, Rose C, Sergent G and L'Herminé C 1999 Am. J. Roentgenol. 1721141

[7] Sreeram K J, Nidhin M, Indumathy R and Nair B U 2008 Bull. Mater. Sci. 3193

[8] Duguet E, Vasseur S, Mornet S, Goglio G, Demourgues A, Portier J et al 2006 Bull. Mater. Sci. 29581

[9] Puntawee S, Theerasilp M, Reabroi S, Saeeng R, Piyachaturawat P, Chairoungdua A et al 2016 Pharm. Dev. Technol. 21 437

[10] Nasongkla N, Shuai X, Ai H, Weinberg B D, Pink J, Boothman D A et al 2004 Angewandte Chemie-International Edition 43 6323

[11] Blanco E, Kessinger C W, Sumer B D and Gao J 2009 Exp. Biol. Med. 234123

[12] Ai H, Flask C, Weinberg B, Shuai X, Pagel M D, Farrell D et al 2005 Adv. Mater. 171949

[13] Huh K M, Lee S C, Cho Y W, Lee J, Jeong J H and Park K 2005 J. Control. Release 10159

[14] Theerasilp M, Chalermpanapun P, Ponlamuangdee K, Sukvanitvichai D and Nasongkla N 2017 RSC Adv. 711158

[15] Sun S, Zeng H, Robinson D B, Raoux S, Rice P M, Wang S X et al 2004 J. Am. Chem. Soc. 126273

[16] Jankova K, Chen X, Kops J and Batsberg W 1998 Macromolecules $\mathbf{3 1} 538$

[17] Crecelius A C, Becer C R, Knop K and Schubert U S $2010 \mathrm{~J}$. Polym. Sci. Pol. Chem. 484375

[18] Kharisov B I, Dias H V R, Kharissova O V, Vázquez A, Peña Y and Gómez I 2014 RSC Adv. 445354

[19] Theerasilp M and Nasongkla N 2013 J. Microencapsul. 30 390

[20] Rutnakornpituk M, Meerod S, Boontha B and Wichai U 2009 Polymer $\mathbf{5 0} 3508$

[21] Ditsch A, Laibinis P E, Wang D I C and Hatton T A 2005 Langmuir 216006

[22] Golas P L, Louie S, Lowry G V, Matyjaszewski K and Tilton R D 2010 Langmuir 2616890

[23] Lim J, Yeap S P, Che H X and Low S C 2013 Nanoscale Res. Lett. 81

[24] Aqil A, Vasseur S, Duguet E, Passirani C, Benoît J P, Roch A et al 2008 Euro. Polym. J. 443191

[25] Shuai X, Merdan T, Schaper A K, Xi F and Kissel T 2004 Bioconjugate Chem. 15441

[26] Astafieva I, Khougaz K and Eisenberg A 1995 Macromolecules 287127

[27] Cheng D, Hong G, Wang W, Yuan R, Ai H, Shen J et al 2011 J. Mater. Chem. 214796

[28] Guardia P, Labarta A and Batlle X 2011 J. Phys. Chem. C 115 390

[29] Nasongkla N, Bey E, Ren J, Ai H, Khemtong C, Guthi J S et al 2006 Nano Lett. 62427

[30] Chen H, Yeh J, Wang L, Khurshid H, Peng N, Wang A Y et al 2010 Nano Res. 3852

[31] Pöselt E, Kloust H, Tromsdorf U, Janschel M, Hahn C, Maßlo C et al 2012 ACS Nano 61619 
[32] Duan H, Kuang M, Wang X, Wang Y A, Mao H and Nie S 2008 J. Phys. Chem. C 1128127

[33] Zhang C, Xie X, Liang S, Li M, Liu Y and Gu H 2012 Magn. Reson. Med. 8996
[34] Arbab A S, Wilson L B, Ashari P, Jordan E K, Lewis B K and Frank J A 2005 NMR Biomed. 18383

[35] Shapiro M G, Atanasijevic T, Faas H, Westmeyer G G and Jasanoff A 2006 Magn. Reson. Imaging 24449 